\title{
Estimation of failure probabilities of linear dynamic systems by importance sampling
}

\author{
ANNA IVANOVA OLSEN and ARVID NAESS \\ Centre for Ships and Ocean Structures (CeSOS), Norwegian University of \\ Science and Technology, NO-7491, Trondheim, Norway \\ e-mail: Anna.Olsen@sintef.no; arvidn@ math.ntnu.no
}

\begin{abstract}
An iterative method for estimating the failure probability for certain time-variant reliability problems has been developed. In the paper, the focus is on the displacement response of a linear oscillator driven by white noise. Failure is then assumed to occur when the displacement response exceeds a critical threshold. The iteration procedure is a two-step method. On the first iteration, a simple control function promoting failure is constructed using the design point weighting principle. After time discretization, two points are chosen to construct a compound deterministic control function. It is based on the time point when the first maximum of the homogenous solution has occurred and on the point at the end of the considered time interval. An importance sampling technique is used in order to estimate the failure probability functional on a set of initial values of state space variables and time. On the second iteration, the concept of optimal control function can be implemented to construct a Markov control which allows much better accuracy in the failure probability estimate than the simple control function. On both iterations, the concept of changing the probability measure by the Girsanov transformation is utilized. As a result the CPU time is substantially reduced compared with the crude Monte Carlo procedure.
\end{abstract}

Keywords. Time-variant reliability; importance sampling; linear dynamic systems.

\section{Introduction}

For many years engineers were mainly concerned with periodic oscillations, i.e. by deterministic periodic functions of time. Requirements of modern industry demand, in some cases, methods which are suitable for the analysis of the responses generated by highly irregular, non-periodic, unrepeatable loads. Thus, the methodology of stochastic dynamics has been developed to handle such demands (Soong \& Grigoriu 1997), and with it the prediction of structural response due to external stochastic oscillating forces has become an important issue in reliability assessment.

This paper is dedicated to Prof R N Iyengar of the Indian Institute of Science on the occasion of his formal retirement. 
For structures subjected to dynamic loads, for instance, due to earthquakes, wind, and ocean waves, the first passage failure is an important characteristic. It occurs when the response reaches for the first time a prescribed tolerance threshold within its duration. In spite of the fact that this problem has received a lot of attention, the calculation of the first passage probability is still one of the most challenging problems in structural reliability. So far, no analytical solution has been obtained even in the case of a linear oscillator. The most widely spread asymptotic solution of this problem is based on the Poisson assumption for the outcrossings from the safe domain and the Rice formula for the outcrossing rate of the response (Lin \& Cai 1995; Soong $\&$ Grigoriu 1997). However, the inaccuracy implied by adopting the Poisson assumption may be quite considerable for practical purposes. On the other hand, Monte Carlo-based methods are found to provide a versatile and robust way for reliability estimation and probabilistic study of such systems. This paper aims at bringing together recent research findings in the application of Monte Carlo methods for reliability assessment, covering fundamental theory, methodology development and applications.

The basic assumption is that performance and design requirements of a dynamical system restrain the acceptable values of the response to the safe domain $D_{S} \in \mathbb{R}^{n}$, where $D_{S}$ is some given set, and the dimension parameter $n$ depends on the problem under study. The probability

$$
p_{S}(T)=P\left\{E_{S}\right\},
$$

where

$$
E_{S}=\left\{\omega: X(t)=X(t, \omega) \in D_{S} \text { for any } 0<t \leq T\right\},
$$

is the probability that the response $X(t)$ will stay inside the safe domain throughout the time interval $(0, T]$. For simplicity it has been assumed that $X(0)=0 . p_{S}(T)$ is referred to as the reliability of the system. Thus the probability of failure $p_{f}(T)$ can be defined as $p_{f}(T)=1-p_{S}(T)$. The response is represented by a continuous stochastic process $X(t, \omega):(0, T] \times \Omega \rightarrow \mathbb{R}^{n}$ defined on a (complete) probability space $(\Omega, \mathcal{F}, P)$ and for the time interval $(0, T]$, where $\Omega$ is a space of elementary events, $\mathcal{F}$ is a $\sigma$-algebra of measurable sets of $\Omega$ and $P: \mathcal{F} \rightarrow[0,1]$ is a probability measure (Øksendal 1998). Thus the probability of failure $p_{f}(T)=1-P\left\{E_{S}\right\}$, where $E_{S} \in \mathcal{F}$ by the assumptions made, is given as

$$
p_{f}(T)=1-\int_{E_{S}} \mathrm{~d} P(\omega)=\int_{\Omega} I[g(X(\cdot, \omega))] \mathrm{d} P(\omega)=E(I[g(X)]),
$$

where $g(X)$ is the limit state function and $I[g(X)]$ is an indicator function defined as follows:

$$
I[g(X)]= \begin{cases}0: & \omega \in E_{S}, \\ 1: & \text { otherwise } .\end{cases}
$$

Thus the mean value estimator of the failure probability by the crude Monte Carlo method is

$$
\widehat{p}_{f}(T)=\frac{1}{N} \sum_{i=1}^{N} I\left[g\left(x^{i}\right)\right],
$$

where $N$ is number of samples, $x^{i}$ is a realization of the process $X_{t}$. The standard deviation of this estimator is given as

$$
\sigma=\left\{\left[\widehat{p}_{f}(T)\left(1-\widehat{p}_{f}(T)\right)\right] / N\right\}^{1 / 2},
$$


hence the standard error of the crude Monte Carlo procedure is $S E \approx 1 /\left(N \widehat{p}_{F}(T)\right)^{1 / 2}$ (Milstein 1995).

Assume that a dynamical system is represented as a Markov diffusion, then the mutually absolutely continuous change of probability measures may be implemented (Chung \& Williams 1990). This change, called the Girsanov transformation (Girsanov 1960; Øksendal 1998), allows the application of a variance reduction procedure, for instance importance sampling, to assess the reliability of the system with a higher convergence rate than the crude Monte Carlo method. Practically the change of measures is accomplished by adding a drift function, called a control function, to the noise term. Milstein (1995) proved that there exists an optimal control function, which leads to zero variance of the functional (3). However it is unattainable, since it depends explicitly on the functional itself.

It is therefore desirable to calculate the approximation of the failure probability functional in order to design a suboptimal control function which allows us to achieve a low variance of the estimator (5). Thus an iterative two-step importance sampling method is presented (Ivanova \& Naess 2004). The procedure uses both concepts of deterministic and Markov control functions. On the first iteration the concept of design point oscillations is used to obtain a first approximation for the failure probability functional (3). On the second stage the Markov control is activated.

The advantage of the proposed procedure is that no special optimization procedures are needed. All the expressions are analytical. Furthermore, for estimating the failure probability tail values of order $10^{-3}$, the CPU time by the importance sampling method is 5 times less than used by the crude Monte Carlo procedure, and this factor is increasing for lower values of the failure probability. The numerical application is presented below. The scope of this paper is restricted to single-degree-of-freedom linear dynamic systems excited by white noise.

\section{Girsanov transformation}

The dynamic response of the system is assumed to be given by an Itô stochastic differential equation (SDE)

$$
\begin{aligned}
\mathrm{d} X(t) & =m(t, X) \mathrm{d} t+\sigma(t, X) \mathrm{d} W(t), \\
X(s) & =x, \quad 0 \leq s \leq t \leq T,
\end{aligned}
$$

where $X(t)=\left(X_{1}(t), X_{2}(t), \ldots, X_{n}(t)\right)^{T} \in \mathbb{R}^{n}$ and $W(t) \in \mathbb{R}$ is a standard Wiener process with respect to the measure $P . m(t, X), \sigma(t, X) \in \mathbb{R}^{n}$ are drift and diffusion coefficients, respectively, which satisfy suitable Lipschitz and growth conditions (Øksendal 1998).

By the Girsanov theorem (Girsanov 1960) the Wiener process $W(t)$ in (7) may be substituted with another stochastic process $\widetilde{W}(t)$ defined as $(0 \leq s \leq t \leq T)$

$$
\widetilde{W}(t)=W(t)-\int_{s}^{t} u(\tau, \omega) \mathrm{d} \tau,
$$

where is $u(\tau, \omega)$ is a real nonanticipative bounded process, for details cf. Øksendal (1998). Define a diffusion

$$
\mathrm{d} \rho_{s}^{t}(u)=-\frac{1}{2} u(t, \omega)^{2} \mathrm{~d} t+u(t, \omega) \mathrm{d} W_{t},
$$

then the following can be stated: 
Theorem 1 (The Girsanov Theorem). Let $W(t)=\left\{w(t, \omega), P, \mathcal{F}_{t}\right\}$ be a Wiener process in $\mathbb{R}_{n}$. Define an Itô process $\widetilde{W}(t)$ of the form

$$
d \widetilde{W}(t)=-u(t, \omega) d t+d W(t), \quad t \leq T<\infty, \widetilde{W}(0)=0 .
$$

Assume that $u(t, \omega)$ satisfies Novikov's condition

$$
E\left[\exp \left(\frac{1}{2} \int_{0}^{T} u^{2}(\tau, \omega) d s\right)\right]<\infty .
$$

Let $\widetilde{P}$ be absolutely continuous with respect to $P$, that is

$$
\widetilde{P}(d \omega)=\exp \rho_{s}^{T}(u) P(d \omega),
$$

where $\rho_{s}^{T}(u)$ is the solution of $(9)$. Moreover assume that $\widetilde{P}(\Omega)=1$.

Then

$$
d \widetilde{X}(t)=m(\widetilde{X}, t) d t+\sigma(\widetilde{X}, t) u(t, \omega) d t+\sigma(\widetilde{X}, t) d \widetilde{W}(t)),
$$

where $\widetilde{W}(t)=\left\{\widetilde{w}(t, \omega), \widetilde{P}, \mathcal{F}_{t}\right\}$ is a Wiener process with respect to a probability measure $\widetilde{P}(\omega)$.

A rigorous proof is given in Øksendal (1998). Thus, $\widetilde{W}(t)$ becomes a standard Wiener process on $(s, T]$ with respect to a new measure $\widetilde{P}$, which is defined below. This transformation of measures may be done according to the Radon-Nikodym theorem (Rudin 1987). By Novikov's condition, the Radon-Nikodym derivative exp $\rho_{s}^{T}(u)=(\mathrm{d} P / \mathrm{d} \widetilde{P})^{-1}$ from (12) is integrable. The Radon-Nikodym theorem then provides us with the measure $\mathrm{d} \widetilde{P}=(\mathrm{d} P / \mathrm{d} \widetilde{P})^{-1} \mathrm{~d} P$ referred to above, and we can write

$$
p_{f}(T ; s, x)=\int_{\Omega} I[g(\widetilde{X})]\left(\frac{\mathrm{d} P}{\mathrm{~d} \widetilde{P}}\right) \mathrm{d} \widetilde{P}=\widetilde{E}\left[I[g(\widetilde{X})]\left(\frac{\mathrm{d} P}{\mathrm{~d} \widetilde{P}}\right)\right],
$$

where $\widetilde{E}$ denotes the mathematical expectation with respect to the measure $\widetilde{P}$. Thence, the Radon-Nikodym derivative can be deducted from the Girsanov theorem

$$
\left(\frac{\mathrm{d} P}{\mathrm{~d} \widetilde{P}}\right)=\exp \left(-\int_{s}^{T} v(\tau, \tilde{X}(\tau)) \mathrm{d} \widetilde{W}(\tau)-\frac{1}{2} \int_{s}^{T} v^{2}(\tau, \tilde{X}(\tau)) \mathrm{d} \tau\right) .
$$

The reliability problem is now considered on the time interval $(s, T]$ with deterministic initial condition $X(s)=x$, and $p_{f}(T ; s, x)$ denotes the associated failure probability. The Monte Carlo estimate of the failure probability based on measure $\widetilde{P}$ is given in Naess (1999),

$$
\widehat{p}_{F}(T ; s, x)=\frac{1}{N} \sum_{i=1}^{N} I\left[g\left(\widetilde{x}^{i}\right)\right]\left(\frac{\mathrm{d} P}{\mathrm{~d} \widetilde{P}}\right)^{i} .
$$

Analysing (3) and (14), it is obvious that the failure probability is independent on the choice of controller, whereas the variance does depend on $v$. Invoking again the theory of stochastic control, it can be shown that there exists an optimal control function (Milstein 1995) for minimizing the functional

$$
J=\widetilde{E}\left[I^{2}[g(\widetilde{X})]\left(\frac{\mathrm{d} P}{\mathrm{~d} \widetilde{P}}\right)^{2}\right] \rightarrow \min
$$


viz.,

$$
v^{*}(s, x)=\frac{1}{p_{f}(T ; s, x)}\left(\sigma(s, x) \cdot \frac{\partial p_{f}(T ; s, x)}{\partial x}\right) .
$$

It can be proved (Milstein 1995) that if $v^{*}(s, x)$ is the optimal control function, then variance of the failure probability estimator is zero, thus the estimator is a deterministic quantity. However, from (18) it follows that the optimal control function depends on the failure probability $p_{f}(T ; s, x)$, which has to be known for all values of the arguments $(s, x) \in$ $(0, T] \times \mathbb{R}^{n}$. But, of course, if the answer is known there is no need to control the system. On the other hand, if the failure probability can be calculated approximately on a suitable finite grid in $(0, T] \times \mathbb{R}^{n}$, i.e. $\widehat{p}_{f}(T ; s, x)$, then it is possible to construct a suboptimal control function $v(s, x)$ such as:

$$
v(s, x)=\frac{1}{\widehat{p}_{F}(T ; s, x)}\left(\sigma(s, x) \cdot \frac{\partial \widehat{p}_{F}(T ; s, x)}{\partial x}\right),
$$

which in general leads to non-zero variance of the estimator, but still may reduce it substantially when $\widehat{p}_{f}(T ; s, x) \approx p_{f}(T ; s, x)$. Thus the goal is to provide such approximation.

\section{Design point oscillations}

In order to evaluate the first approximation of the failure probability functional (3) the concept of design point oscillations has been implemented. The idea was first introduced by Newton (1994), followed by Tanaka (1998). Further, the method was extended to nonlinear and multidimensional dynamic systems by Au \& Beck (2001) and Macke \& Bucher (2003). The procedure showed fast convergence to the crude Monte Carlo estimates. However, the main drawback of this method is that the design point oscillations are not generally available in the analytical form, thus special optimization techniques are required.

Assume that $X(t)=\left(X_{1}(t), \ldots, X_{n}(t)\right)^{T}$ is a solution of the SDE (7) on the interval $(s, T]$. In order to use the Monte Carlo method, independent realizations of this solution have to be obtained. Assume also that the equidistant time discretization mesh with step size $\Delta t=(T-s) / M$ is given by

$$
s=t_{0} \leq t_{1} \leq \cdots \leq t_{i} \leq \cdots \leq t_{M}=T \quad t_{i}=s+i \Delta t .
$$

The SDE (7) can be approximated by a difference scheme, for instance, the EulerMaruyama scheme

$$
\begin{aligned}
X_{k}\left(t_{i+1}\right) & =X_{k}\left(t_{i}\right)+m_{k}\left(t_{i}, X\right) \Delta t+\sigma_{k}\left(t_{i}, X\right) \Delta W\left(t_{i}\right), \\
X_{k}\left(t_{0}\right) & =x_{k},
\end{aligned}
$$

where the increments of the Wiener process are given as

$$
\Delta W\left(t_{i}\right)=U_{i} \sqrt{\Delta t} \quad(i=0, \ldots, M-1)
$$

with $U_{i}$ being mutually independent Gaussian $N(0,1)$-variables. 
As it is mentioned above, the safe domain is described in the terms of the limit state function $g(X)$. Due to discretization the number of random variables is truncated to a finite quantity. Thus, the limit state surface at a time $t_{j}$ may be expressed through the normalized variables as:

$$
g\left(X\left(t_{j}\right)\right)=g\left(X\left(U_{0}, \ldots, U_{j-1}\right)\right)=g_{U}\left(U_{0}, \ldots, U_{(j-1)}\right)=0 .
$$

The design point associated with the time $t_{j}$ is the point on the failure surface $g_{U}\left(u_{0}, \ldots, u_{j-1}\right)=0$ closest to the origin in $\mathbf{R}^{j}$. Denote this point by $\left\{u_{0}^{j}, \ldots, u_{j-1}^{j}\right\}$. The minimal distance is then given by the relation

$$
\beta\left(t_{j}\right)=\left[\sum_{i=0}^{j-1}\left(u_{i}^{j}\right)^{2}\right]^{1 / 2},
$$

where $\beta\left(t_{j}\right)$ is referred to as the design point index. The design point determines what is denoted as the design point oscillations, leading most likely to a failure event at the time $t_{j}$. In some cases, when a single failure time $t^{*}$ is dominant, for instance, in a case of fatigue fracture (Tanaka 1999), an accurate estimate of the failure probability might be obtained by employing the control corresponding to the minimal design point index

$$
\beta\left(t^{*}\right)=\min _{s<t \leq T} \beta(t)
$$

For oscillatory systems, this approach leads to a substantial underestimation of the failure probability (Naess \& Skaug 2000). Due to the system characteristics certain magnitudes contributes periodically to the first passage probability. To take this effect into account all the contributions should be exploited. Thus, as it is shown by Macke \& Bucher (2003), those effects can be weighted by the coefficients

$$
w_{j}=\left[\Phi\left(-\beta\left(t_{j}\right)\right)\right] /\left[\sum_{i=1}^{M} \Phi\left(-\beta\left(t_{i}\right)\right)\right],
$$

where $j=1, \ldots, M$ and $\Phi(\cdot)$ is a cumulative standard Gaussian distribution.

Consider now the controlled system (13) in the discrete form

$$
\begin{aligned}
\tilde{X}_{k}\left(t_{i+1}\right)= & \tilde{X}_{k}\left(t_{i}\right)+m_{k}\left(t_{i}, \tilde{X}\right) \Delta t+\sigma_{k}\left(t_{i}, \tilde{X}\right) v\left(t_{i}, \tilde{X}\right) \Delta t \\
& +\sigma_{k}\left(t_{i}, \widetilde{X}\right) \Delta \widetilde{W}\left(t_{i}\right),
\end{aligned}
$$

where the controls are given as:

$$
v\left(t_{i}, \tilde{X}\right)=v\left(t_{i}\right)=(1 / \sqrt{\Delta t}) u_{i}^{j}, \quad(i<j, j=1, \ldots, M) .
$$

Hence the recurrent expression for the design point oscillations is obtained. Nevertheless, the numerical evaluation can be a challenging task due to the large number of Gaussian variables and nonlinearities. However, for the linear case, an analytical expression for the design point oscillations is available. This fact is used to obtain the numerical approximation of the failure probability functional. The numerical example on implementation is shown below. 


\section{Numerical illustration}

The considered numerical example is based on the fact that a very wide class of engineering systems can be modelled, with good approximation, in terms of linear differential equations of motion, if the amplitude of motion is relatively small. For instance, behaviour of a linear spring-mass system excited by the white noise $N(t)$ follows the second order linear differential equation:

$$
\begin{aligned}
& \ddot{X}(t)+2 \xi \omega_{0} \dot{X}(t)+\omega_{0}^{2} X(t)=\gamma N(t), \\
& X(s)=x, \quad \dot{X}(s)=\dot{x},
\end{aligned}
$$

where $X(t)$ is a displacement, $\omega_{0}$ is a normalized free vibration angular frequency, $\xi$ is a damping ratio, $\gamma$ is an intensity of the noise term. For the sake of generality, the initial conditions are assumed non-zero. The considered safe domain is given by,

$$
D_{S}=\left\{(x, \dot{x}): x<x_{c}, \dot{x} \in \mathbb{R}\right\},
$$

where $x_{c}$ is a prescribed critical threshold for any $0 \leq s \leq t \leq T$.

Under standard assumptions (30) can be written as an $\operatorname{SDE}(7)$. Let $X_{1}=X$ and $X_{2}=\dot{X}$, then (30) is given in matrix form as

$$
d \hat{X}(t)=A \hat{X}(t) d t+\gamma b d W(t),
$$

where $W(t)$ is a standard scalar Wiener process (Soong \& Grigoriu 1997) and

$$
\hat{X}=\left[\begin{array}{l}
X_{1} \\
X_{2}
\end{array}\right], \quad A=\left[\begin{array}{cc}
0 & 1 \\
-2 \xi \omega_{0} & -\omega_{0}^{2}
\end{array}\right], \quad b=\left[\begin{array}{l}
0 \\
1
\end{array}\right] .
$$

Hereby the main characteristics of this system should be mentioned (Soong \& Grigoriu 1997). The approximate variance of the displacement is given

$$
\sigma_{X}^{2}(t) \cong \frac{\pi G_{0}}{4 \xi \omega_{0}^{3}}\left(1-\frac{1}{\omega_{d}^{2}} e^{-2 \xi \omega_{0} t}\left(\omega_{d}^{2}+2 \xi^{2} \omega_{0}^{2} \sin ^{2} \omega_{d} t+\xi \omega_{0} \omega_{d} \sin 2 \omega_{d} t\right)\right),
$$

where $G_{0}=\gamma^{2} / \pi$ is a one-sided spectral density of the white noise excitations, $\omega_{d}=$ $\omega_{0}\left(1-\xi^{2}\right)^{1 / 2}$ is a damped free oscillation frequency. Hence, the stationary variance of the response is obtained for $t \rightarrow \infty$,

$$
\sigma_{X}^{2}=\left(\pi G_{0}\right) /\left(4 \xi \omega_{0}^{3}\right) .
$$

Furthermore, in the stationary regime, the mean values of displacement $E[X(t)]=0$ and velocity $E[\dot{X}(t)]=0$. The stationary spectral density of the response can be obtained straightforwardly as

$$
S_{X}(\omega)=\left[G_{0} / 2\right] /\left[\left(\omega_{0}^{2}-\omega^{2}\right)^{2}+\left(2 \xi \omega_{0} \omega\right)^{2}\right] .
$$

In figures $1 \mathrm{a}$ and $\mathrm{b}$ the spectral density and variance of displacement $X(t)$ are illustrated respectively. In the figure, the time axis is normalized by $T_{n}=2 \pi / \omega_{0}$, the natural period of the free undamped oscillations. Since the field of interest in this project is the excitations 

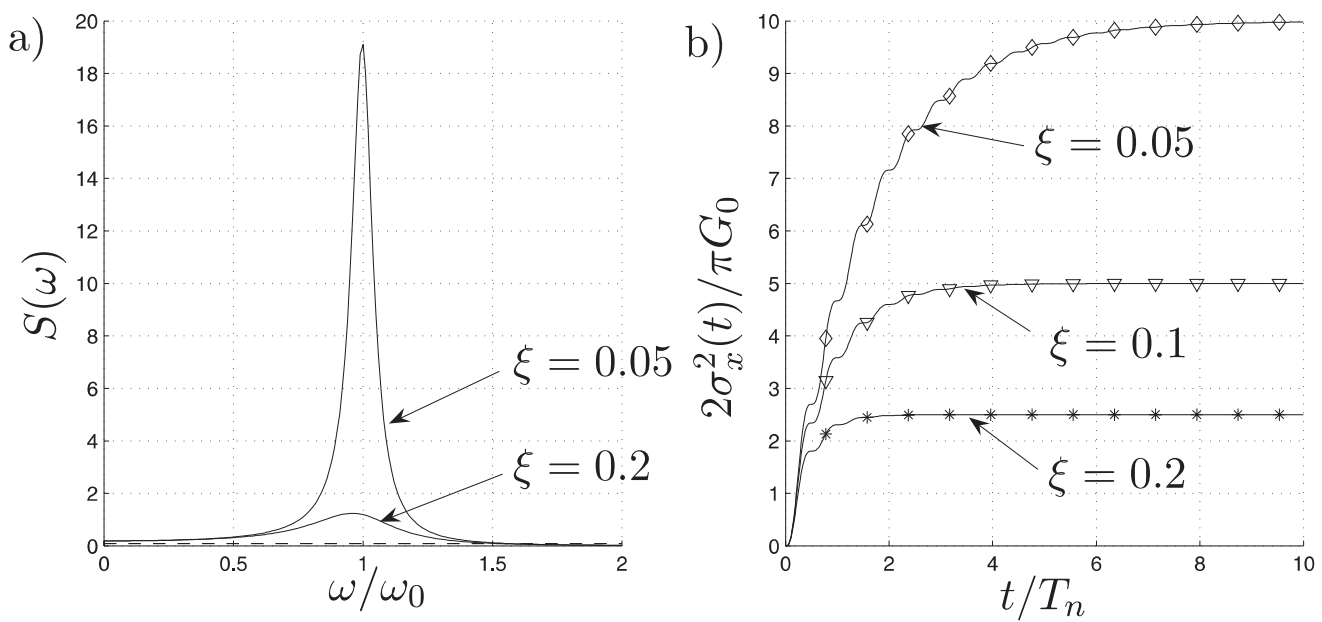

Figure 1. (a) Power spectral density and (b) variance of the displacement $X(t)$ for different values of damping ratio $\xi$.

of non-stationary transient nature or at least of "short duration", all the characteristics of the system are chosen such as its response still depends on initial values.

Furthermore, (30) has an explicit analytical solution

$$
X(t)=F(t-s, x, \dot{x})+\gamma \int_{s}^{t} h(t-\tau) d W(\tau),
$$

where

$$
h(t)=\left(1 / \omega_{d}\right) e^{-\xi \omega_{0} t} \sin \omega_{d} t
$$

is an impulse response function and,

$$
F(t, x, \dot{x})=\left(1 / \omega_{d}\right) e^{-\xi \omega_{0} t}\left(\left(\xi \omega_{0} x+\dot{x}\right) \sin \omega_{d} t+\omega_{d} x \cos \omega_{d} t\right),
$$

is an homogenous solution for the displacement $X(t)$. Thus there exists an explicit relationship between the displacement and the Wiener process. Using the forward Euler approximation, (38) can be approximated by the difference scheme:

$$
X\left(t_{j}\right) \approx F\left(t_{j}-s, x, \dot{x}\right)+\gamma \sum_{i=0}^{j-1} h_{j i} U_{i} \sqrt{\Delta t},
$$

where $s=t_{0}<\cdots<t_{j}<\cdots<t_{M}=T, t_{j}=t_{0}+j \cdot \Delta t, \Delta t=(T-s) / M$, $h_{j i}=h((j-i) \cdot \Delta t), U_{i} \sqrt{\Delta t}=W\left(t_{i+1}\right)-W\left(t_{i}\right)$ are the increments of the Wiener process. $U_{i}$ are independent standard Gaussian variables with zero mean and unit standard deviation.

Considering the given safe domain (32) the failure function at the time point $t_{j}$ takes the form

$$
g\left(\hat{X}\left(t_{j}\right)\right)=x_{c}-X\left(t_{j}\right), \quad \hat{X}=\left(X\left(t_{j}\right), \dot{X}\left(t_{j}\right)\right)^{T} .
$$


Using the discrete representation of the solution path (41) the limit state function (42) can be mapped to the finite normalized Gaussian space

$$
g\left(\hat{X}\left(t_{j}\right)\right)=x_{c}-X\left(t_{j}\right) \approx x_{c}-F\left(t_{j}-s, x, \dot{x}\right)-\sqrt{\gamma} \sum_{i=0}^{j-1} h_{j i} U_{i} \sqrt{\Delta t}=g_{U}(U) .
$$

The limit state surface $g_{U}(u)=0, u=\left(u_{0}, \ldots, u_{j-1}\right)^{T}$, gives the expression for the design point oscillations corresponding to a failure at a time point $t_{j}$

$$
u_{i}=\left\{\left[x_{c}-F\left(t_{j}-s, x, \dot{x}\right)\right] / \gamma \sum_{i=0}^{j-1} h_{j i}^{2} \sqrt{\Delta t}\right\} \cdot h_{j i}, i=0, \ldots, j-1,
$$

and, correspondingly, the design point index is

$$
\beta\left(t_{j}\right)=\left\{\sum_{i=0}^{j-1} u_{i}^{2}\right\}^{1 / 2}
$$

To achieve variance reduction the Wiener process $W(t)$ is replaced by $\widetilde{W}(t)$ as explained in $\S 2$., (33) takes on the form

$$
\mathrm{d} \tilde{\hat{X}}(t)=A \tilde{\hat{X}}(t) \mathrm{d} t+\gamma b v(t, \tilde{\hat{X}}(t)) \mathrm{d} t+\gamma b \mathrm{~d} \widetilde{W}(t) .
$$

On the first iteration, the approximation of the failure probability is obtained by using a deterministic open loop control, $v(t, \hat{X}(t))=v(t)$. Then for the transformed system (46) the function (29) is used. Thus

$$
v_{i}=\left\{\left[x_{c}-F\left(t_{j}-s, x, \dot{x}\right)\right] / \gamma \sum_{i=0}^{j-1} h_{j i}^{2} \Delta t\right\} \cdot h_{j i}, i=0, \ldots, j-1,
$$

where if $\Delta t \rightarrow 0$ then (47) can be written in the integral form (Naess \& Skaug 2000)

$$
v(t)=\left\{\left[x_{c}-X_{h}\left(t_{j}-s\right)\right] / \gamma \int_{s}^{t_{j}} h^{2}\left(t_{j}-\tau\right) \mathrm{d} \tau\right\} \cdot h\left(t_{j}-t\right), s \leq t \leq t_{j} .
$$

To obtain the first approximation of the failure probability it is proposed to apply design point oscillations which cause failure at the end of the considered time interval $T$. That is natural because most of the samples approach the critical threshold at the end of response duration, especially samples started at zero initial conditions. The design point index in this case has minimum at the end point $T$,

$$
\beta^{*}=\beta(T)=\min _{s<t \leq T} \beta(t) .
$$

However, considering non-zero initial conditions the system may be more prone to failure at the beginning of the time interval because of the system initial energy. To compensate this effect it was proposed to use design point oscillations which lead to a failure event at the time of the first maximum of the homogenous solution $F(t-s, x, \dot{x})$. In figure 2 the design point 


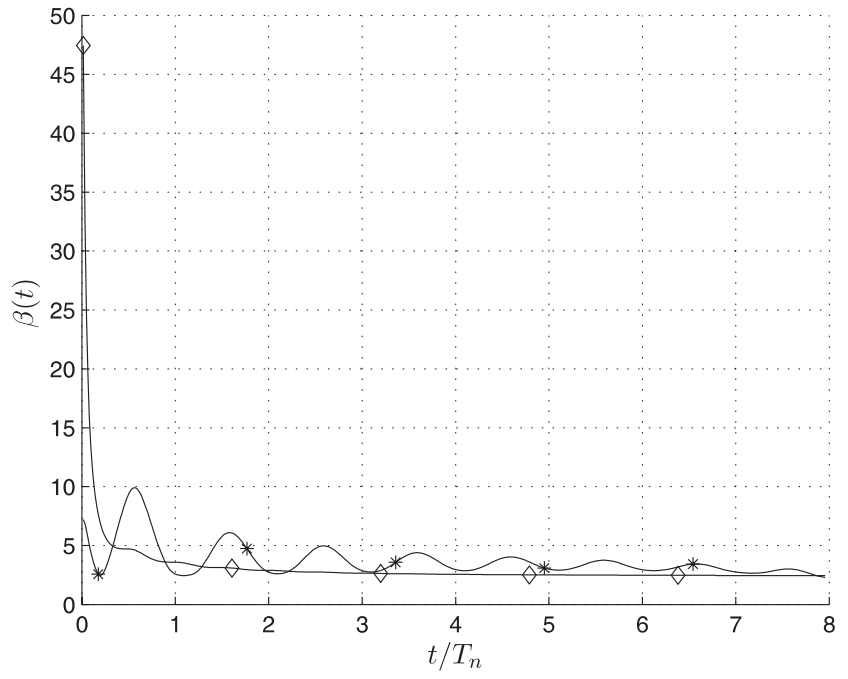

Figure 2. The design point index vs time; $(\diamond)$ - zero initial values, $(*)-$ non-zero initial values.

index is illustrated for system with zero and non-zero initial values. The curve, corresponding to the non-zero initial values and marked with asterisks $(*)$, also has the global minimum at the end point $T$. Nevertheless, as it can be seen, the first local minimum near the origin has a magnitude comparable with the global value. Hence, the failure near this point will give a significant contribution to the failure probability. Taking into account these features, two design times $t_{(1)}=T$ and $t_{(2)}=t^{\max }$ are used, weighted according to their importance $w_{(j)}$ (27).

So the simulations using the open loop control (48) are performed on a grid of initial values $(x, \dot{x})$ and for different values of a starting time point $s$. Hence the first approximation of the failure probability functional $\widehat{p}_{f}(T ; s, x, \dot{x})$ is obtained. Figure 3 shows a smoothed logarithm of the failure probability functional for $s=0$ in the state space. On the second

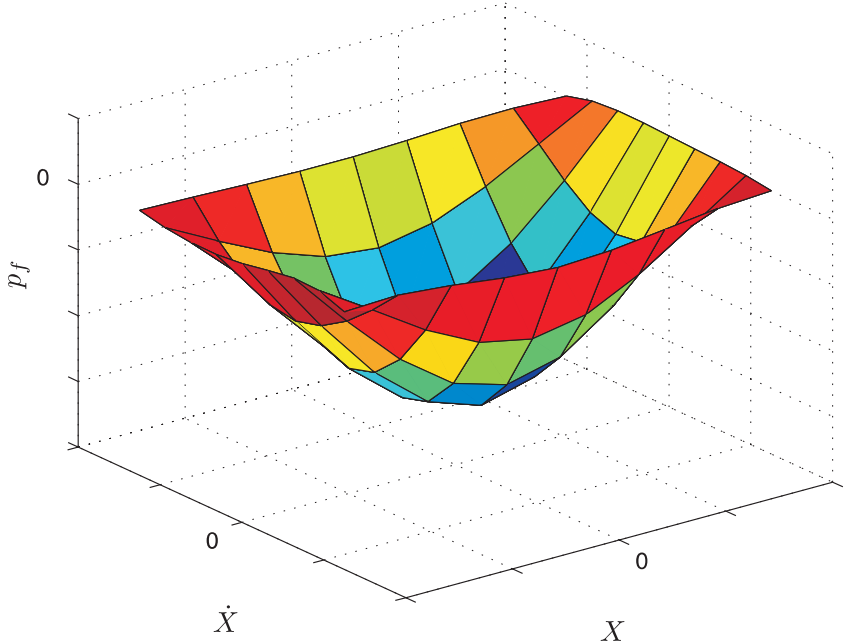

Figure 3. Results of the first iteration - approximate failure probability in $\mathbb{R}^{2}=[X \times \dot{X}]$ smoothed by B-splines. 
iteration during the numerical integration at each time point, the value of the optimal Markov control function is taken from 3D data calculated on the first iteration. The value of this approximate control function at each time step is sought explicitly as the point depending on the state space variables on the previous time step $v_{i}=v\left(t_{i-1}, x_{1}\left(t_{i-1}\right), x_{2}\left(t_{i-1}\right)\right)$. After the sample path crosses a threshold $x_{c}$, the control function is set to zero. The results of the study are presented in the next section.

\section{Results}

The following cases were considered during the study of the method: case 1 is a narrow-band oscillatory system, where the damping ratio is $\xi=0.05$, and case 2 is a broad-band one with $\xi=0 \cdot 2$. The first model might correspond to the oscillations of an aircraft structure and the second one might represent the vibration of some rubber elements in a machine. For both cases the free oscillation frequency $\omega_{0}=1$ and the intensity of external excitations $\gamma=(0 \cdot 3)^{1 / 2}$ are the same.

For the narrow-band case the transient period is about $8 \cdot T_{n}$, whereas for the broad-band case the transient period is about 1.5. $T_{n}$. The sample paths for both cases are shown in figures 4 and 5 correspondingly. The ordinate on all figures is normalized by $\sigma_{X}(36)$. The (a)-plots on both figures present the samples of the displacement $X(t)$ and the velocity $\dot{X}(t)$ of the original system (30). Due to the choice of parameter $\omega_{0}=1$ the displacement and velocity have the same order of magnitude. These processes oscillate normally inside the stripe $\left[-2 \sigma_{X}, 2 \sigma_{X}\right]$.

The (b)-plots show the paths of the system (46) controlled by the open-loop control $v(t)$ (48). Here the design time chosen is $t_{(1)}=T$. Thus the design point oscillations force the failure event near the end of the considered time interval. The control is unaware of the system state and follows the only condition that $X(T) \geq x_{c}$.

The samples of the linear system controlled by the Markov control $v(t, X, \dot{X})(19)$ are in the (c)-plots. In this case the controller is following the sample path pursuing the aim that $X(t) \geq x_{c}$ for $t \in(0, T]$. Thence the first passage is likely to occur anywhere on this interval. The smoothness of $v(t, X, \dot{X})$ is a matter of accuracy of the first approximation.

a)

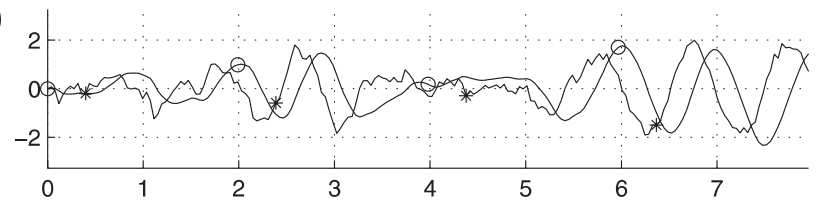

b)

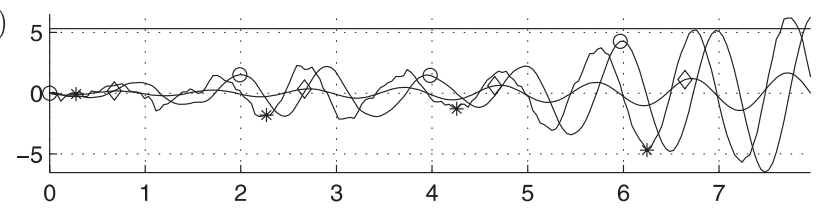

c)

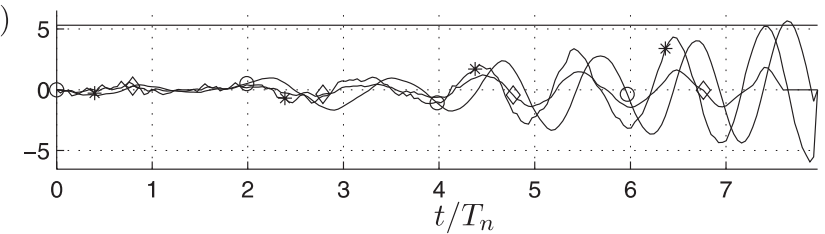

Figure 4. Case 1: (a) (o) $x_{1}(t)$ and $(*) x_{2}(t)$ for the original equation (30), (b) (o) $x_{1}(t)$ and $(*)$ $x_{2}(t)$ for the controlled equation (46) with deterministic control $(\diamond)$ $v(t)$ and (c) with Markov control $(\diamond) v\left(t, x_{1}, x_{2}\right)$; critical threshold $x_{c} / \sigma_{X}=5 \cdot 3$. 
a)

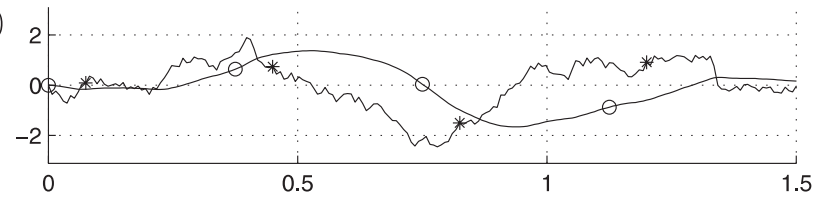

b)

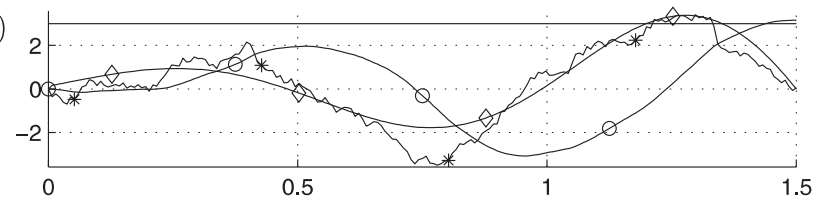

c)

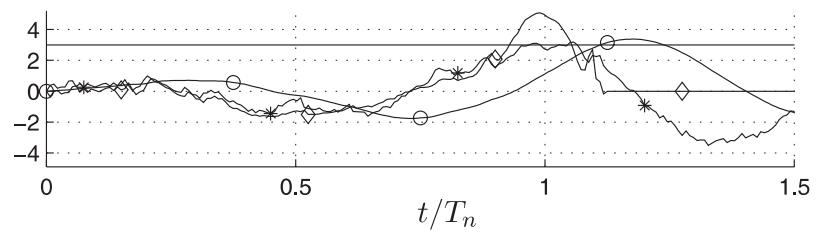

Figure 5. Case 2: (a) (o) $x_{1}(t)$ and $(*) x_{2}(t)$ for the original equation (30), (b) (o) $x_{1}(t)$ and $(*)$ $x_{2}(t)$ for the controlled equation (46) with deterministic control $(\diamond)$ $v(t)$ and (c) with Markov control $(\diamond) v\left(t, x_{1}, x_{2}\right)$; critical threshold $x_{c} / \sigma_{X}=3$.

In figures 6 and 7 the results of numerical experiments vs critical threshold are shown for both cases correspondingly. The critical threshold is also normalized by $\sigma_{X}$ (36).

The test results were compared with estimates calculated by the crude Monte Carlo method. Those are obtained with different number of samples $\left(>10^{3}\right)$ by the convergency criterium that standard error $(S E)$ equals $0 \cdot 05$, otherwise, if $S E$ has not converged to $0 \cdot 05$, the number of samples is $N=10^{6}$. Whereas $N$ used by the importance sampling procedure in the two steps is $n_{x} \times n_{\dot{x}} \times n_{t} \times n_{1}+n_{2}$, where $n_{x}$ is the number of grid points in $x$-direction, $n_{\dot{x}}$ is in $\dot{x}$-direction, $n_{t}$ is in time, $n_{1}$ is number of samples simulated with the set of initial values $(x, \dot{x}, s)$ and $n_{2}$ is number of samples on the second iteration. The results from the first iteration is obtained with $n_{1}=10 \ldots 100$ and two open-loop controls aimed at the failure

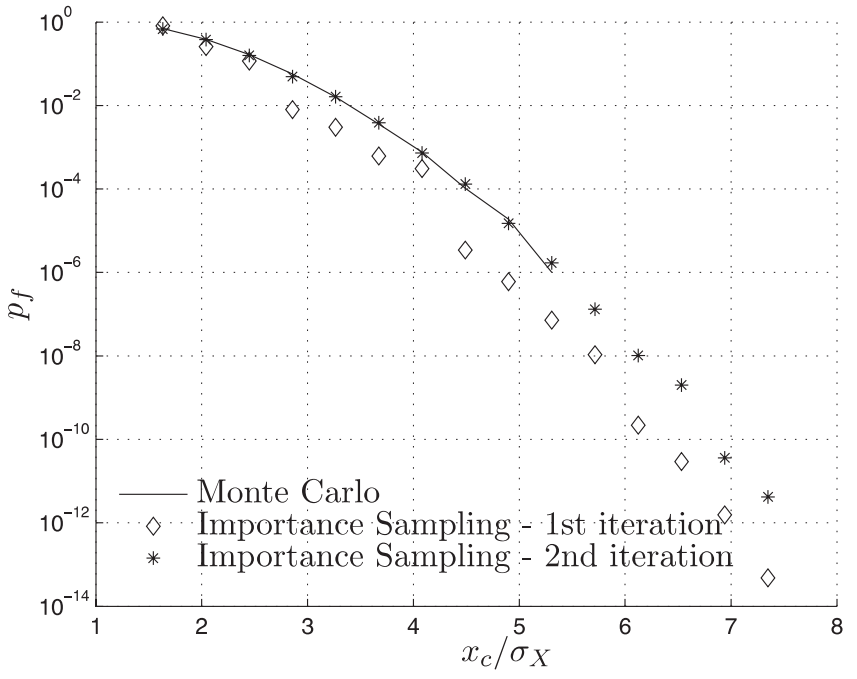

Figure 6. Case 1: failure probability vs critical threshold. 


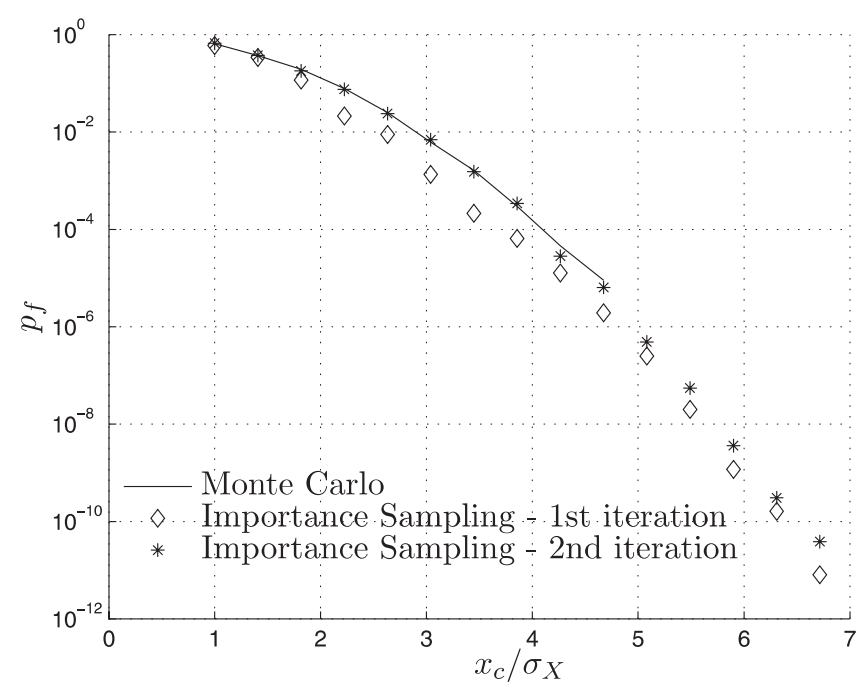

Figure 7. Case 2: failure probability vs critical threshold.

events at $t_{(1)}=T$ and $t_{(2)}=T / 2$. Due to the low number of samples the first approximation values have standard error of order $0 \cdot 1-0 \cdot 8$.

The standard error of the importance sampling estimates for whole procedure has converged to 0.05 with the number of samples used on the second iteration between $n_{2}=50 \ldots 5 \cdot 10^{3}$. This does not affect the total calculation time very much, because most of the computational burden is associated with the first step. For instance, for the value of the failure probability $p_{f}=7 \cdot 7 \cdot 10^{-3}$ estimated with the standard error $S E=0.05$ the crude Monte Carlo method used $N=51497$ samples and $31 \mathrm{~s}$ calculation time, whereas the iterative importance sampling achieved this value with $N=4531\left(n_{1}=10\right)$ and $3 \mathrm{~s}$ CPU time with the same confidence.

In figure 8 , the failure probability vs damping ratio $\xi$ is presented. The considered time interval is $T=1 \cdot 5 \cdot T_{n}$ which is less than the transition zone of the system with the parameter $\xi=0.2$. This experiment shows that the procedure is applicable to the wide range of system parameters causing from narrow-band to wide-band processes.

Figure 9 presents the results the failure probability versus time. As in the previous figures for time great than $T>0 \cdot 5 \cdot T_{n}$, the iterative importance sampling has better convergence to a Monte Carlo result than importance sampling with the design point oscillations. Whereas for the short time intervals $T \leq 0 \cdot 5 \cdot T_{n}$ both methods require approximately the same number of samples to achieve the chosen standard error value. This explains why for time less than half a period the system behaves as non-oscillatory and the principle of the one dominant failure point is valid.

\section{Conclusion}

An iterative two-step importance sampling procedure has been presented to estimate the failure probability of structural dynamic systems modelled as Itô stochastic differential equations. The linear oscillator excited by the white noise was used as an example.

The study showed a fast convergence of the failure probability estimates in the proposed importance sampling procedure to the results of the crude Monte Carlo method until this method breaks down. 


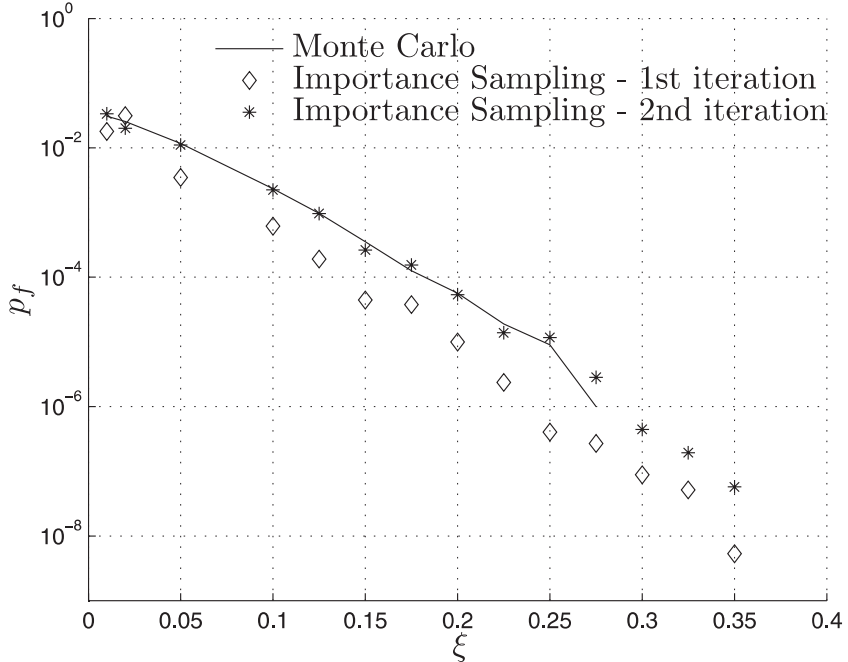

Figure 8. Failure probability vs damping ratio.

As one of the advantages of the scheme it might be mentioned that it is possible to calculate robust values of low probabilities of failure of the order $10^{-3}$ and less. Moreover, no special software is required for the computations. All expressions for design point oscillations can be found analytically. Furthermore, it was shown that the procedure is insensitive to the system parameters, i.e. to whether the response is narrow or broad banded.

The CPU time for the importance sampling procedure compared with the crude Monte Carlo is at least 5 times less for the failure probability values of order $10^{-3}$, whereas for tail values lower than $10^{-3}$ the speed up is even more drastic.

Further work is in progress to adjust the iterative importance sampling procedure for nonlinear problems (for instance, Duffing oscillator) and dynamic systems driven by non-white

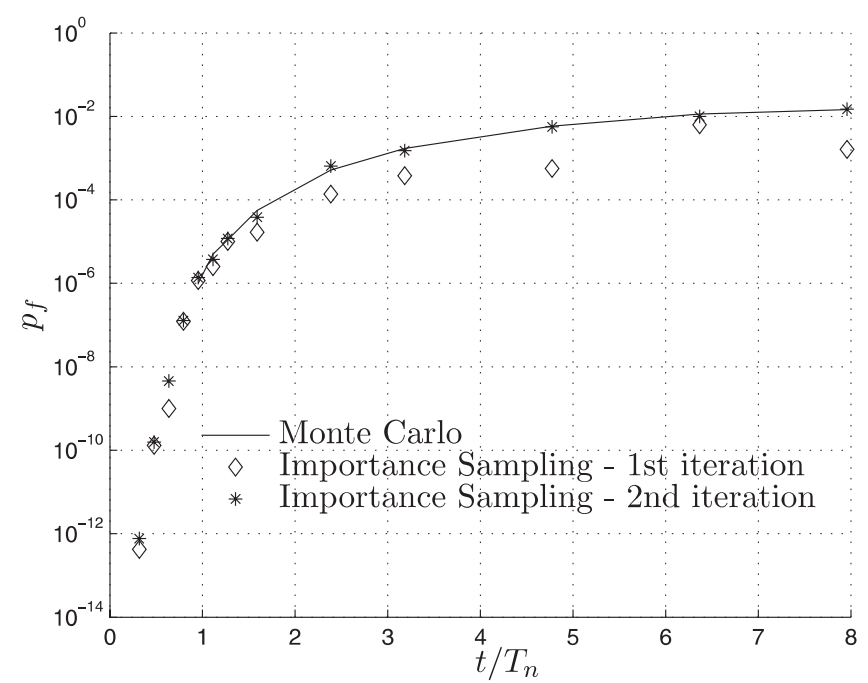

Figure 9. Case 1: failure probability vs time; critical threshold $x_{c} / \sigma_{X}=3 \cdot 3$. 
excitations. It is proposed to use on the first iteration an auxiliary linear system which has the same upcrossing rate as the original system.

This project is financially supported by the Research Council of Norway. The authors gratefully acknowledge this support.

\section{References}

Au S K, Beck J L 2001 First excursion probabilities for linear systems by very efficient importance sampling. Probab. Eng. Mech. 16: 193-207

Chung K L, Williams R J 1990 Introduction to stochastic integration 2nd edn (Boston: Birkhäuser)

Girsanov I V 1960 On transforming a certain class of stochastic processes by absolutely continuous substitution of measures. Theor. Probab. Appl. 5: 285-301

Ivanova A, Naess A 2004 Importance sampling for dynamic systems by approximate calculation of the optimal control function. Proc. 4th Int. Conf. on Mathematical Methods in Reliability, Sante Fe, NM, USA (Singapore: World Scientific)

Lin Y K, Cai G Q 1995 Probabilistic structural dynamics. advanced theory and applications. (MCGraw-Hill)

Macke M, Bucher C 2003 Importance sampling for randomly excited dynamical systems. J. Sound Vibr. 268: 269-290

Milstein G N 1995 Numerical integration of stochastic differential equations (Dordrecht: Kluwer Academic)

Naess A 1999 Comments on importance sampling for time variant reliability problems. Stochastic Struct. Dyn. 4: 197-202

Naess A, Skaug C 2000 Importance sampling for dynamical systems. In Proc. ICASP 8 Conference (eds) R E Melchers, M G Stewart (Rotterdam: A A Balkema) pp 749-755

Newton N J 1994 Variance reduction for simulated diffusions. SIAM J. Appl. Math. 54: 1780-1805

Øksendal B 1998 Stochastic differential equations: An introduction with application (5th edn) (Berlin: Springer)

Olsen A I, Naess A 2004 Importance sampling for dynamic systems by approximate calculation of the optimal control function. In Modern statistical and mathematical methods in reliability of series on quality, reliability and engineering statistics (eds) A Wilson, N Limnios, S Keller-McNulty, Y Armijo (Singapore: World Scientific) 10: 339-352

Rudin W 1987 Real and complex analysis (New York: McGraw-Hill)

Soong T T, Grigoriu M 1997 Random vibration of mechanical and structural systems (Englewood Cliffs, NJ: Prentice-Hall)

Tanaka H 1998 Application of an importance sampling method to time-dependent system reliability analyses using the girsanov transformation. Proceedings of ICOSSAR'97 - The 7th International Conference on Structural Safety and Reliability (eds) N Shiraishi, M Shinozuka, Y K Wen (Rotterdam: A A Balkema) 1: 411-418

Tanaka H 1999 Importance sampling simulation for a stochastic fatigue crack growth model. In Proc. ICASP 8 Conference (eds) R E Melchers, M G Stewart (Rotterdam: A A Balkema) pp 749-755 